\title{
Some classes of Cesàro-type difference sequences over $n$-normed spaces
}

Hemen Dutta*

"Correspondence:

hemen_dutta08@rediffmail.com

Department of Mathematics,

Gauhati University, Guwahati,

Assam 781014, India

\begin{abstract}
In this paper, we introduce some Cesàro-type difference sequences spaces defined over a real linear $n$-normed space and investigate the spaces for completeness under suitable $n$-norm in each case. Relevant relations among the classes of sequences are examined. We also introduce the notion of $n-B K$-spaces and show that the spaces can be made an $n$-BK-space under certain condition. Further, we compute the Köthe-Toeplitz duals of the spaces, wherever possible within the scope of the research of this article.
\end{abstract}

MSC: 40A05; 46A20; 46D05; 46A45; 46E30

Keywords: Cesàro summability; difference sequences; $n$-normed spaces; completeness; Köthe-Toeplitz dual

\section{Introduction}

The studies of linear transformation on sequence spaces are called summability. The earliest idea of summability theory was perhaps contained in a letter written by Leibnitz to Wolf (1713), in which the sum of the oscillatory series 1-1+1-1+1 - - - as given by Leibnitz was $\frac{1}{2}$. Studies on sequence space were further extended through summability theory. Summability theory, or in short summability, is the theory of the assignment of limits, which is fundamental in analysis, function theory, topology and functional analysis.

Throughout $w, \ell_{\infty}, \ell_{p}, l_{1}, c$ and $c_{0}$ denote the spaces of all, bounded, $p$-absolutely summable, absolutely summable, convergent and null sequences $x=\left(x_{k}\right)$ with complex terms, respectively.

The zero element of a normed linear space (n.l.s.) is denoted by $\theta$. A complete n.l.s. is called a Banach space.

$\ell_{p}(0<p<\infty)$ denotes the space of all complex sequences such that $\sum_{k}\left|x_{k}\right|^{p}<\infty$, called as the space of $p$-absolutely summable sequences. The space $\ell_{p}$ for $p \geq 1$ is complete under the norm defined by $\|x\|=\left(\sum_{k}\left|x_{k}\right|^{p}\right)^{1 / p}$.

For $0<p<1, \ell_{p}$ is a complete $p$-normed space, $p$-normed by $\|x\|=\sum_{k=1}^{\infty}\left|x_{k}\right|^{p}$.

A $B K$-space (introduced by Zeller [1]) $(X,\|\cdot\|)$ is a Banach space of complex sequences $x=\left(x_{k}\right)$, in which the co-ordinate maps are continuous, that is, $\left|x_{k}^{n}-x_{k}\right| \rightarrow 0$, whenever $\left\|x^{n}-x\right\| \rightarrow 0$ as $n \rightarrow \infty$, where $x^{n}=\left(x_{k}^{n}\right)$ for all $n \in N$ and $x=\left(x_{k}\right)$.

Let $(X,\|\cdot\|)$ be a normed linear space, and $\lambda$ is a scalar-valued sequence space, then the vector-valued sequence space or $X$-valued sequence space $\lambda(X)$ is defined as $\lambda(X)=\left\{\left(x_{k}\right)\right.$ : $x_{k} \in X$ for all $k \in N$ and $\left.\left\|x_{k}\right\| \in \lambda\right\}$.

@2013 Dutta; licensee Springer. This is an Open Access article distributed under the terms of the Creative Commons Attribution License (http://creativecommons.org/licenses/by/2.0), which permits unrestricted use, distribution, and reproduction in any medium, provided the original work is properly cited. 
Clearly, $\lambda(X)$ is a linear space under coordinate wise addition and scalar multiplication over the field of scalars of $X$.

Similarly, if $X$ is a Banach space, then $\ell_{p}(X)(1=p<\infty)$ is a Banach space with the norm given by $\|x\|=\left(\sum_{k=1}^{\infty}\left\|x_{k}\right\|^{p}\right)^{\frac{1}{p}}$.

The Cesàro means (also called Cesàro averages) of sequence $\left\{a_{n}\right\}$ are the terms of sequence $\left\{c_{n}\right\}$, where $c_{n}=\sum_{i=1}^{n} a_{n}$ is the arithmetic mean of the first $n$ elements of $\left\{a_{n}\right\}$. This concept is named after Ernesto Cesàro. It is known that if $\left\{a_{n}\right\}$ converges to $l$, then $\left\{a_{n}\right\}$ also converges to the same limit. This means that the operation of taking Cesàro means preserves convergent sequences and their limits. This is the basis of taking Cesàro means as a summability method in the theory of divergent sequences. If the sequence of the Cesàro means is convergent, the series is said to be Cesàro summable. There are certainly many examples, for which the sequence of Cesàro means converges, but the original sequence does not. For example, sequence $\left\{a_{n}\right\}=\left\{(-1)^{n}\right\}$ which is Cesàro summable to 0 .

The Cesàro sequence spaces

$$
\operatorname{Ces}_{p}=\left\{x=\left(x_{k}\right):\|x\|_{p}=\left(\sum_{n=1}^{\infty} \frac{1}{n} \sum_{k=1}^{n}\left|x_{k}\right|^{p}\right)^{\frac{1}{p}}<\infty, 1 \leq p<\infty\right\}
$$

and

$$
\operatorname{Ces}_{8}=\left\{x=\left(x_{k}\right):\|x\|_{\infty}=\sup _{n} \frac{1}{n} \sum_{k=1}^{n}\left|x_{k}\right|<\infty\right\}
$$

have been introduced and studied by Shiue [2], and it was observed that $\ell_{p} \subset \operatorname{Ces}_{p}$ $(1<p<8)$ is strict, although it does not hold for $p=1$.

Nag and Lee [3] defined and studied the Cesàro sequence space $X_{p}$ of non-absolute-type as follows:

$$
X_{p}=\left\{x=\left(x_{k}\right):\|x\|_{p}=\left(\sum_{n=1}^{\infty}\left|\frac{1}{n} \sum_{k=1}^{n} x_{k}\right|^{p}\right)^{\frac{1}{p}}<\infty, 1 \leq p<\infty\right\}
$$

and

$$
X_{\infty}=\left\{x=\left(x_{k}\right):\|x\|_{\infty}=\sup _{n}\left|\frac{1}{n} \sum_{k=1}^{n} x_{k}\right|<\infty\right\} .
$$

The inclusion $\operatorname{Ces}_{p} \subset X_{p}, 1 \leq p<\infty$ is strict.

Orhan [4] defined and studied the Cesàro difference sequence spaces $X_{p}(\Delta)$ and $X_{\infty}(\Delta)$ by replacing $x=\left(x_{k}\right)$ with $\Delta x=\left(\Delta x_{k}\right)=\left(x_{k}-x_{k+1}\right), k=1,2, \ldots$ and showed that for $1 \leq$ $p<\infty$, the inclusions $X_{p} \subset X_{p}(\Delta)$ and $X_{\infty} \subset X_{\infty}(\Delta)$ are strict. In fact, Orhan [4] used $C_{p}$ instead of $X_{p}(\Delta)$ and $C_{\infty}$ instead of $X_{\infty}(\Delta)$.

Further, Orhan [4] defined and studied the following sequence spaces:

$$
O_{p}(\Delta)=\left\{x=\left(x_{k}\right): \sum_{n=1}^{\infty}\left(\frac{1}{n} \sum_{k=1}^{n}\left|\Delta x_{k}\right|\right)^{p}<\infty, 1 \leq p<\infty\right\}
$$


and

$$
O_{\infty}(\Delta)=\left\{x=\left(x_{k}\right): \sup _{n \geq 1} \frac{1}{n} \sum_{k=1}^{n}\left|\Delta x_{k}\right|<\infty\right\} .
$$

He showed that for $1 \leq p<\infty$, the inclusions $O_{p}(\Delta) \subset X_{p}(\Delta)$ and $\operatorname{Ces}_{p} \subset O_{p}(\Delta)$ are strict.

Mursaleen et al. [5] defined and studied the Cesàro difference sequence space $X_{p}\left(\Delta^{2}\right)$, i.e.,

$$
X_{p}\left(\Delta^{2}\right)=\left\{x=\left(x_{k}\right): \sum_{n=1}^{\infty}\left|\frac{1}{n} \sum_{k=1}^{n} \Delta^{2} x_{k}\right|^{p}<\infty, 1 \leq p<\infty\right\},
$$

and

$$
X_{\infty}\left(\Delta^{2}\right)=\left\{x=\left(x_{k}\right): \sup _{n \geq 1}\left|\frac{1}{n} \sum_{k=1}^{n} \Delta^{2} x_{k}\right|<\infty\right\},
$$

where $\Delta^{2} x_{k}=\Delta x_{k}-\Delta x_{k+1}$.

For uniformity of the literature, henceforth, we shall write $C_{p}$ instead of $X_{p}$ and $C_{\infty}$ instead of $X_{\infty}$.

For some useful works on Cesàro-type summable spaces, we refer to [6-8].

Let $E$ and $F$ be two sequence spaces. Then the $F$ dual of $E$ is defined as $E^{F}=\left\{\left(x_{k}\right) \in w\right.$ : $\left(x_{k} y_{k}\right) \in F$ for all $\left.\left(y_{k}\right) \in E\right\}$.

For $F=l_{1}$, the dual is termed as $\alpha$-dual (Köthe-Toeplitz dual) of $E$ and denoted by $E^{\alpha}$. If $X \subset Y$, then $Y^{\alpha} \subset X^{\alpha}$.

For initial and useful works on the notion of Köthe-Toeplitz duals, we refer to [9-12].

Let $n \in N$ and $X$ be a real vector space of dimension $d$, where $n \leq d$. A real-valued function $\|\cdot, \ldots, \cdot\|$ on $X^{n}$ satisfying the following four conditions:

(N1) $\left\|x_{1}, x_{2}, \ldots, x_{n}\right\|=0$ if and only if $x_{1}, x_{2}, \ldots, x_{n}$ are linearly dependent,

(N2) $\left\|x_{1}, x_{2}, \ldots, x_{n}\right\|$ is invariant under permutation,

(N3) $\left\|\alpha x_{1}, x_{2}, \ldots, x_{n}\right\|=|\alpha|\left\|x_{1}, x_{2}, \ldots, x_{n}\right\|$, for any $\alpha \in R$,

(N4) $\left\|x+x^{\prime}, x_{2}, \ldots, x_{n}\right\| \leq\left\|x, x_{2}, \ldots, x_{n}\right\|+\left\|x^{\prime}, x_{2}, \ldots, x_{n}\right\|$

is called an $n$-norm on $X$, and the pair $(X,\|\cdot, \ldots, \cdot\|)$ is called an $n$-normed space.

A trivial example of an $n$-normed space is $X=R^{n}$ equipped with the following Euclidean n-norm:

$$
\left\|x_{1}, x_{2}, \ldots, x_{n}\right\|_{E}=\operatorname{abs}\left(\left|\begin{array}{ccc}
x_{11} & \cdots & x_{1 n} \\
\vdots & \ddots & \vdots \\
x_{n 1} & \cdots & x_{n n}
\end{array}\right|\right)
$$

where $x_{i}=\left(x_{i 1}, \ldots, x_{i n}\right) \in R^{n}$ for each $i=1,2, \ldots, n$.

Gunawan [13] showed how we can actually define an $n$-inner product and, accordingly, an $n$-norm on any inner product space provided the dimension is sufficiently large as follows: 
Let $n \in N$ and $(X,\langle\cdot, \cdot\rangle)$ be a real inner product space of dimension $d=n$. Define the following function $\langle\cdot, \ldots, \cdot \mid \cdot, \cdot\rangle$ on $X \times \cdots \times X(n+1$ factors $)$ by

$$
\left\langle x_{1}, \ldots, x_{n-1} \mid y, z\right\rangle=\left|\begin{array}{cccc}
\left\langle x_{1}, x_{1}\right\rangle & \cdots & \left\langle x_{1}, x_{n-1}\right\rangle & \left\langle x_{1}, z\right\rangle \\
\vdots & \ddots & \vdots & \vdots \\
\left\langle x_{n-1}, x_{1}\right\rangle & \cdots & \left\langle x_{n-1}, x_{n-1}\right\rangle & \left\langle x_{n-1}, z\right\rangle \\
\left\langle y, x_{1}\right\rangle & \cdots & \left\langle y, x_{n-1}\right\rangle & \langle y, z\rangle
\end{array}\right| .
$$

Then one may check that this function satisfies the following five properties:

(I1) $\left\langle x_{1}, \ldots, x_{n-1} \mid x_{n}, x_{n}\right\rangle=0 ;\left\langle x_{1}, \ldots, x_{n-1} \mid x_{n}, x_{n}\right\rangle=0$ if and only if $x_{1}, \ldots, x_{n}$ are linearly dependent;

(I2) $\left\langle x_{1}, \ldots, x_{n-1} \mid x_{n}, x_{n}\right\rangle=\left\langle x_{i_{1}}, \ldots, x_{i_{n-1}} \mid x_{i_{n}}, x_{i_{n}}\right\rangle$ for every permutation $\left(i_{1}, \ldots, i_{n}\right)$ of $(1, \ldots, n)$

(I3) $\left\langle x_{1}, \ldots, x_{n-1} \mid y, z\right\rangle=\left\langle x_{1}, \ldots, x_{n-1} \mid z, y\right\rangle$;

(I4) $\left\langle x_{1}, \ldots, x_{n-1} \mid y, \alpha z\right\rangle=\alpha\left\langle x_{1}, \ldots, x_{n-1} \mid y, z\right\rangle$;

(I5) $\left\langle x_{1}, \ldots, x_{n-1} \mid y, z+z^{\prime}\right\rangle=\left\langle x_{1}, \ldots, x_{n-1} \mid y, z\right\rangle+\left\langle x_{1}, \ldots, x_{n-1} \mid y, z^{\prime}\right\rangle$.

Accordingly, we can define $\|\cdot, \ldots, \cdot\|$ on $X \times \cdots \times X$ ( $n$ factors) by

$$
\left\|x_{1}, \ldots, x_{n}\right\|=\left\langle x_{1}, \ldots, x_{n-1} \mid x_{n}, x_{n}\right\rangle^{1 / 2},
$$

that is,

$$
\left\|x_{1}, \ldots, x_{n}\right\|=\left|\begin{array}{ccc}
\left\langle x_{1}, x_{1}\right\rangle & \cdots & \left\langle x_{1}, x_{n}\right\rangle \\
\vdots & \ddots & \vdots \\
\left\langle x_{n}, x_{1}\right\rangle & \cdots & \left\langle x_{n}, x_{n}\right\rangle
\end{array}\right|^{\frac{1}{2}}
$$

For $n=1$, we know that $\|\cdot\|$ is a norm, while for $n=2,\|\cdot, \cdot\|$ defines a 2 -norm. Note further that for $n=1,\left\|x_{1}\right\|$ gives the length of $x_{1}$, while for $n=2,\left\|x_{1}, x_{2}\right\|$ represents the area of the parallelogram spanned by $x_{1}$ and $x_{2}$. For $n=3$ and $X=R^{3}$, one may observe that $\left\|x_{1}, x_{2}, x_{3}\right\|$ is nothing but the volume of the parallelepiped spanned by $x_{1}, x_{2}$ and $x_{3}$, that is, $\left\|x_{1}, x_{2}, x_{3}\right\|=\left|x_{1} \cdot\left(x_{2} \times x_{3}\right)\right|$.

Sequence $\left(x_{k}\right)$ in an $n$-normed space $(X,\|\cdot, \ldots, \cdot\|)$ is said to converge to some $L \in X$ in the $n$-norm if

$$
\lim _{k \rightarrow \infty}\left\|x_{k}-L, u_{2}, \ldots, u_{n}\right\|=0 \quad \text { for every } u_{2}, \ldots, u_{n} \in X
$$

Sequence $\left(x_{k}\right)$ in an $n$-normed space $(X,\|\cdot, \ldots, \cdot\|)$ is said to be Cauchy with respect to the $n$-norm if

$$
\lim _{k, l \rightarrow \infty}\left\|x_{k}-x_{l}, u_{2}, \ldots, u_{n}\right\|=0 \quad \text { for every } u_{2}, \ldots, u_{n} \in X
$$

If every Cauchy sequence in $X$ converges to some $L \in X$, then $X$ is said to be complete with respect to the $n$-norm. Any complete $n$-normed space is said to be $n$-Banach space.

For some relevant works on 2-normed structure and its extension to $n(\geq 2)$-normed structure and subsequent applications, one may refer to [14-30]. 
The notion of difference sequence space was introduced by Kizmaz [31], who studied the difference sequence spaces $\ell_{\infty}(\Delta), c(\Delta)$ and $c_{0}(\Delta)$. The notion was further generalized by Et and Colak [32] by introducing the spaces $\ell_{\infty}\left(\Delta^{m}\right), c\left(\Delta^{m}\right)$ and $c_{0}\left(\Delta^{m}\right)$, where $n$ is a non-negative integer. In general, we have the following definition of difference sequence spaces.

Let $m$ be non-negative integers, then for $Z$, a given sequence space, we have

$$
Z\left(\Delta^{m}\right)=\left\{x=\left(x_{k}\right) \in w:\left(\Delta^{m} x_{k}\right) \in Z\right\}
$$

where $\Delta^{m} x=\left(\Delta^{m} x_{k}\right)=\left(\Delta^{m-1} x_{k}-\Delta^{m-1} x_{k+1}\right)$ and $\Delta^{0} x_{k}=x_{k}$ for all $k \in N$, which is equivalent to the following binomial representation:

$$
\Delta^{m} x_{k}=\sum_{v=0}^{m}(-1)^{v}\left(\begin{array}{c}
m \\
v
\end{array}\right) x_{k+v} .
$$

\section{Definitions and preliminaries}

Let $(X,\|\cdot, \ldots, \cdot\|)$ be an $n$-normed real linear space, and $w(n-X)$ denotes $X$-valued sequence space. Let $m$ be a non-negative integer and $1 \leq p<\infty$, then we introduce the following sequence spaces for every non zero $z_{1}, \ldots, z_{n-1} \in X$ :

$$
\begin{aligned}
& C_{p}\left(\Delta^{m},\|\cdot, \cdot, \ldots, \cdot\|\right) \\
& \quad=\left\{\left(x_{k}\right) \in w(n-X): \sum_{i=1}^{\infty}\left(\left\|\frac{1}{i} \sum_{k=1}^{i} \Delta^{m} x_{k}, z_{1}, \ldots, z_{n-1}\right\|\right)^{p}<\infty\right\}, \\
& C_{\infty}\left(\Delta^{m},\|\cdot, \ldots, \cdot\|\right) \\
& \quad=\left\{\left(x_{k}\right) \in w(n-X): \sup _{i}\left(\left\|\frac{1}{i} \sum_{k=1}^{i} \Delta^{m} x_{k}, z_{1}, \ldots, z_{n-1}\right\|\right)<\infty\right\}, \\
& \ell_{p}\left(\Delta^{m},\|\cdot, \ldots, \cdot\|\right) \\
& \quad=\left\{\left(x_{k}\right) \in w(n-X): \sum_{k=1}^{\infty}\left(\left\|\Delta^{m} x_{k}, z_{1}, \ldots, z_{n-1}\right\|\right)^{p}<\infty\right\}, \\
& O_{p}\left(\Delta^{m},\|\cdot, \ldots, \cdot\|\right) \quad \\
& \quad=\left\{\left(x_{k}\right) \in w(n-X): \sum_{i=1}^{\infty}\left(\frac{1}{i} \sum_{k=1}^{i}\left(\left\|\Delta^{m} x_{k}, z_{1}, \ldots, z_{n-1}\right\|\right)\right)^{p}<\infty\right\}, \\
& O_{\infty}\left(\Delta^{m},\|\cdot, \ldots, \cdot\|\right) \quad \\
& \quad=\left\{\left(x_{k}\right) \in w(n-X): \sup _{i}\left(\frac{1}{i} \sum_{k=1}^{i}\left\|\Delta^{m} x_{k}, z_{1}, \ldots, z_{n-1}\right\|\right)<\infty\right\} .
\end{aligned}
$$

We procure the following result, which will help us in establishing the results of this article.

Lemma 2.1 (Tripathy, Esi and Tripathy [33])

(a) Let $1 \leq p<\infty$. Then 
(i) The space $C_{p}$ is a Banach space, normed by

$$
\|x\|=\left(\sum_{i=1}^{\infty}\left|\frac{1}{i} \sum_{k=1}^{i} x_{k}\right|^{p}\right)^{\frac{1}{p}} .
$$

(ii) The space $O_{p}$ is a Banach space, normed by

$$
\|x\|=\left(\sum_{i=1}^{\infty} \frac{1}{i} \sum_{k=1}^{i}\left|x_{k}\right|^{p}\right)^{\frac{1}{p}} .
$$

(iii) The space $\ell_{p}$ is a Banach space, normed by

$$
\|x\|=\left(\sum_{k=1}^{\infty}\left|x_{k}\right|^{p}\right)^{\frac{1}{p}} .
$$

(b) (i) The space $C_{\infty}$ is a Banach space, normed by

$$
\|x\|=\sup _{i}\left|\frac{1}{i} \sum_{k=1}^{i} x_{k}\right| .
$$

(ii) The space $\mathrm{O}_{\infty}$ is a Banach space, normed by

$$
\|x\|=\sup _{i} \frac{1}{i} \sum_{k=1}^{i}\left|x_{k}\right| .
$$

Definition 2.1 An $n$-BK-space $(X,\|\cdot, \ldots, \cdot\|)$ is an $n$-Banach space of real sequences $x=$ $\left(x_{k}\right)$ in which the co-ordinate maps are continuous.

\section{Construction of $n$-norms and relevant properties}

In this section, we construct $n$-norms on the introduced spaces of previous section and investigate the spaces for completeness and some relations among them. The proof of the following result is a routine verification.

Proposition 3.1 The classes of sequences $C_{p}\left(\Delta^{m},\|\cdot, \ldots, \cdot\|\right), O_{p}\left(\Delta^{m},\|\cdot, \ldots, \cdot\|\right), \ell_{p}\left(\Delta^{m}\right.$, $\|\cdot, \ldots, \cdot\|), C_{\infty}\left(\Delta^{m},\|\cdot, \ldots, \cdot\|\right)$ and $O_{\infty}\left(\Delta^{m},\|\cdot, \ldots, \cdot\|\right)$ for $1 \leq p<\infty$ are linear spaces over the field of reals.

\section{Theorem 3.2}

(a) Let $1 \leq p<\infty$, and the base space $X$ is an $n$-Banach space. Then

(i) The space $C_{p}\left(\Delta^{m},\|\cdot, \ldots, \cdot\|\right)$ is an $n$-Banach space, $n$-normed by $\left\|x^{1}, x^{2}, \ldots, x^{n}\right\|_{C_{P}}^{\Delta^{m}}=0$ if $x^{1}, x^{2}, \ldots, x^{n}$ are linearly dependent and $=\sum_{k=1}^{m}\left\|x_{k}, z_{1}, \ldots, z_{n-1}\right\|+\left(\sum_{i=1}^{\infty}\left\|\frac{1}{i} \sum_{k=1}^{i} \Delta^{m} x_{k}, z_{1}, \ldots, z_{n-1}\right\|^{p}\right)^{\frac{1}{p}}$ for every $z_{1}, \ldots, z_{n-1} \in X$ if $x^{1}, x^{2}, \ldots, x^{n}$ are linearly independent.

(ii) The space $O_{p}\left(\Delta^{m},\|\cdot, \ldots, \cdot\|\right)$ is an $n$-Banach space, $n$-normed by $\left\|x^{1}, x^{2}, \ldots, x^{n}\right\|_{O_{P}}^{\Delta^{m}}=0$ if $x^{1}, x^{2}, \ldots, x^{n}$ are linearly dependent and 
$=\sum_{k=1}^{m}\left\|x_{k}, z_{1}, \ldots, z_{n-1}\right\|+\left(\sum_{i=1}^{\infty} \frac{1}{i} \sum_{k=1}^{i}\left\|\Delta^{m} x_{k}, z_{1}, \ldots, z_{n-1}\right\|^{p}\right)^{\frac{1}{p}}$ for every $z_{1}, \ldots, z_{n-1} \in X$ if $x^{1}, x^{2}, \ldots, x^{n}$ are linearly independent.

(iii) The space $\ell_{p}\left(\Delta^{m},\|\cdot, \ldots, \cdot\|\right)$ is an $n$-Banach space, $n$-normed by $\left\|x^{1}, x^{2}, \ldots, x^{n}\right\|_{\ell_{p}}^{\Delta^{m}}=0$, if $x^{1}, x^{2}, \ldots, x^{n}$ are linearly dependent and $=\sum_{k=1}^{m}\left\|x_{k}, z_{1}, \ldots, z_{n-1}\right\|+\left(\sum_{k=1}^{\infty}\left\|\Delta^{m} x_{k}, z_{1}, \ldots, z_{n-1}\right\|^{p}\right)^{\frac{1}{p}}$ for every $z_{1}, \ldots, z_{n-1} \in X$ if $x^{1}, x^{2}, \ldots, x^{n}$ are linearly independent.

(b) (i) The space $C_{\infty}\left(\Delta^{m},\|\cdot, \ldots, \cdot\|\right)$ is an $n$-Banach space, $n$-normed by $\left\|x^{1}, x^{2}, \ldots, x^{n}\right\|_{C_{\infty}}^{\Delta^{m}}=0$ if $x^{1}, x^{2}, \ldots, x^{n}$ are linearly dependent and $=\sum_{k=1}^{m}\left\|x_{k}, z_{1}, \ldots, z_{n-1}\right\|+\sup _{i}\left\|\frac{1}{i} \sum_{k=1}^{i} \Delta^{m} x_{k}, z_{1}, \ldots, z_{n-1}\right\|$ for every $z_{1}, \ldots, z_{n-1} \in X$ if $x^{1}, x^{2}, \ldots, x^{n}$ are linearly independent.

(ii) The space $O_{\infty}\left(\Delta^{m},\|\cdot, \ldots, \cdot\|\right)$ is an $n$-Banach space, $n$-normed by $\left\|x^{1}, x^{2}, \ldots, x^{n}\right\|_{C_{\infty}}^{\Delta^{m}}=0$ if $x^{1}, x^{2}, \ldots, x^{n}$ are linearly dependent and $=\sum_{k=1}^{m}\left\|x_{k}, z_{1}, \ldots, z_{n-1}\right\|+\sup _{i} \frac{1}{i} \sum_{k=1}^{i}\left\|\Delta^{m} x_{k}, z_{1}, \ldots, z_{n-1}\right\|$ for every $z_{1}, \ldots, z_{n-1} \in X$ if $x^{1}, x^{2}, \ldots, x^{n}$ are linearly independent.

Proof It is a routine verification that the spaces $C_{p}\left(\Delta^{m},\|\cdot, \ldots, \cdot\|\right), O_{p}\left(\Delta^{m},\|\cdot, \ldots, \cdot\|\right)$, $\ell_{p}\left(\Delta^{m},\|\cdot, \ldots, \cdot\|\right), C_{\infty}\left(\Delta^{m},\|\cdot, \ldots, \cdot\|\right)$ and $O_{\infty}\left(\Delta^{m},\|\cdot, \ldots, \cdot\|\right)$ are $n$-normed spaces under the $n$-norm, defined as above.

Here, we prove the completeness for the space $C_{\infty}\left(\Delta^{m},\|\cdot, \ldots, \cdot\|\right)$, and for the other spaces, it will follow on applying similar arguments.

Let $\left(x^{s}\right)_{s=1}^{\infty}$ be a Cauchy sequence in $C_{\infty}\left(\Delta^{m},\|\cdot, \ldots, \cdot\|\right)$, where $x^{s}=\left(x_{i}^{s}\right)=\left(x_{1}^{s}, x_{2}^{s}, \ldots\right) \in$ $C_{\infty}\left(\Delta^{m},\|\cdot, \ldots, \cdot\|\right)$ for each $s \in N$. Let $\varepsilon>0$ be given. Then there exists a positive integer $n_{0}$ such that $\left\|x^{s}-x^{t}, u^{2}, \ldots, u^{n}\right\|_{C_{\infty}}^{\Delta^{m}}<\varepsilon$ for all $s, t \geq n_{0}$ and for every $u^{2}, \ldots, u^{n}$ in $C_{\infty}\left(\Delta^{m},\|\cdot, \ldots, \cdot\|\right)$.

$$
\Rightarrow \quad \sum_{k=1}^{m}\left\|x_{k}^{s}-x_{k}^{t}, z_{1}, \ldots, z_{n-1}\right\|+\sup _{i}\left\|\frac{1}{i} \sum_{k=1}^{i} \Delta^{m}\left(x_{k}^{s}-x_{k}^{t}\right), z_{1}, \ldots, z_{n-1}\right\|<\varepsilon
$$

for all $s, t \geq n_{0}$ and for every $z_{1}, \ldots, z_{n-1} \in X$.

$$
\Rightarrow \quad \sum_{k=1}^{m}\left\|x_{k}^{s}-x_{k}^{t}, z_{1}, \ldots, z_{n-1}\right\|<\varepsilon \text { and } \sup _{i}\left\|\frac{1}{i} \sum_{k=1}^{i} \Delta^{m}\left(x_{k}^{s}-x_{k}^{t}\right), z_{1}, \ldots, z_{n-1}\right\|<\varepsilon
$$

for all $s, t \geq n_{0}$ and for every $z_{1}, \ldots, z_{n-1} \in X$.

Hence $\left\|x_{k}^{s}-x_{k}^{t}, z_{1}, \ldots, z_{n-1}\right\|<\varepsilon$ for all $k=1,2, \ldots, m$ and for every $z_{1}, \ldots, z_{n-1} \in X$.

$\Rightarrow\left(x_{k}^{s}\right)$ is a Cauchy sequence for all $k=1,2, \ldots, m$ in $X$, an $n$-Banach space.

Hence, $\left(x_{k}^{s}\right)$ converges in $X$ for all $k=1,2, \ldots, m$. Let $\lim _{s \rightarrow \infty} x_{k}^{s}=x_{k}$ for all $k=1,2, \ldots, m$.

Next, we have

$$
\sup _{i}\left\|\frac{1}{i} \sum_{k=1}^{i} \Delta^{m}\left(x_{k}^{s}-x_{k}^{t}\right), z_{1}, \ldots, z_{n-1}\right\|<\varepsilon
$$

for all $s, t \geq n_{0}$ and for every $z_{1}, \ldots, z_{n-1} \in X$.

This implies that for every $z_{1}, \ldots, z_{n-1} \in X$

$$
\left\|\frac{1}{i} \sum_{k=1}^{i} \Delta^{m}\left(x_{k}^{s}-x_{k}^{t}\right), z_{1}, \ldots, z_{n-1}\right\|<\varepsilon \quad \text { for all } s, t \geq n_{0} \text { and } i \in N .
$$


$\Rightarrow\left(\Delta^{m} x_{k}^{s}\right)$ is a Cauchy sequence in $C_{\infty}(\|\cdot, \ldots, \cdot\|)$, which is complete (it is easy to check that $C_{\infty}(\|\cdot, \ldots, \cdot\|)$ is complete).

Hence, $\left(\Delta^{n} x_{k}^{s}\right)$ converges for each $k \in N$. Let $\lim _{s \rightarrow \infty} \Delta_{m}^{n} x_{k}^{s}=y_{k}$ for each $k \in N$.

Let $k=1$, we have

$$
\lim _{s \rightarrow \infty} \Delta^{m} x_{1}^{s}=\lim _{s \rightarrow \infty} \sum_{v=0}^{m}(-1)^{v}\left(\begin{array}{l}
m \\
v
\end{array}\right) x_{1+v}=y_{1}
$$

We have

$$
\lim _{s \rightarrow \infty} x_{k}^{s}=x_{k}, \quad \text { for } k=1+v \text {, for } v=1,2, \ldots, m-1 .
$$

Thus, from (3.2.1) and (3.2.2), we have that $\lim _{s \rightarrow \infty} x_{1+m}^{s}$ exists. Let $\lim _{s \rightarrow \infty} x_{1+m}^{s}=x_{1+m}$. Proceeding in this way inductively, we have that $\lim _{s \rightarrow \infty} x_{k}^{s}=x_{k}$ exists for each $k \in N$.

Now, for every $z_{1}, \ldots, z_{n-1} \in X$

$$
\lim _{t} \sum_{k=1}^{m}\left\|x_{k}^{s}-x_{k}^{t}, z_{1}, \ldots, z_{n-1}\right\|=\sum_{k=1}^{m}\left\|x_{k}^{s}-x_{k}, z_{1}, \ldots, z_{n-1}\right\|<\varepsilon \quad \text { for all } s \geq n_{0} \text {. }
$$

Again, using the continuity of $n$-norm, we find that for every $z_{1}, \ldots, z_{n-1} \in X$

$$
\left\|\frac{1}{i} \sum_{k=1}^{i} \Delta^{m} x_{k}^{s}-\lim _{t \rightarrow \infty} \Delta^{m} x_{k}^{t}, z_{1}, \ldots, z_{n-1}\right\|<\varepsilon \quad \text { for all } s \geq n_{0} \text { and } i \in N
$$

Hence, for every $z_{1}, \ldots, z_{n-1} \in X$

$$
\sup _{i}\left\|\frac{1}{i} \sum_{k=1}^{i} \Delta^{m} x_{k}^{s}-\Delta^{m} x_{k}, z_{1}, \ldots, z_{n-1}\right\|<\varepsilon \quad \text { for all } s \geq n_{0} .
$$

Thus, for every $u^{2}, \ldots, u^{n}$ in $C_{\infty}\left(\Delta^{m},\|\cdot, \ldots, \cdot\|\right)$

$$
\left\|x^{s}-x, u^{2}, \ldots, u^{n}\right\|_{C_{\infty}}^{\Delta^{m}}<2 \varepsilon \quad \text { for all } s \geq n_{0} .
$$

Hence, $\left(x^{s}-x\right) \in C_{\infty}\left(\Delta^{m},\|\cdot, \ldots, \cdot\|\right)$. Since $C_{\infty}\left(\Delta^{m},\|\cdot, \ldots, \cdot\|\right)$ is a linear space, so we have for all $s \geq n_{0}$,

$$
x=x^{s}-\left(x^{s}-x\right) \in C_{\infty}\left(\Delta^{m},\|\cdot, \ldots, \cdot\|\right) .
$$

Hence, $C_{\infty}\left(\Delta^{m},\|\cdot, \ldots, \cdot\|\right)$ is complete and as such is an $n$-Banach space.

Corollary 3.3 The spaces $C_{p}\left(\Delta^{m},\|\cdot, \ldots, \cdot\|\right), O_{p}\left(\Delta^{m},\|\cdot, \ldots, \cdot\|\right), \ell_{p}\left(\Delta^{m},\|\cdot, \ldots, \cdot\|\right), C_{\infty}\left(\Delta^{m}\right.$, $\|\cdot, \ldots, \cdot\|)$ and $O_{\infty}\left(\Delta^{m},\|\cdot, \ldots, \cdot\|\right)$ for $1 \leq p<\infty$ are $n$-BK-spaces if the base space $X$ is an n-Banach space.

Proof The proof is obvious in view of the previous theorem.

Theorem $3.4 Z\left(\Delta^{m-1},\|\cdot, \ldots, \cdot\|\right) \subset Z\left(\Delta^{m},\|\cdot, \ldots, \cdot\|\right)$ (in general $Z\left(\Delta^{i},\|\cdot, \ldots, \cdot\|\right) \subset Z\left(\Delta^{m}\right.$, $\|\cdot, \ldots, \cdot\|)$, for $i=1,2, \ldots, m-1)$ for $Z=C_{p}, O_{p}, \ell_{p}, C_{\infty}$ and $O_{\infty}$. 
Proof Here, we prove the result for $Z=C_{p}$ and for the other results, it will follow on applying similar arguments.

Let $x=\left(x_{k}\right) \in C_{p}\left(\Delta^{m-1},\|\cdot, \ldots, \cdot\|\right), 1 \leq p<\infty$. Then for every non zero $z_{1}, \ldots, z_{n-1} \in X$,

$$
\sum_{i=1}^{\infty}\left(\left\|\frac{1}{i} \sum_{k=1}^{i} \Delta^{m-1} x_{k}, z_{1}, \ldots, z_{n-1}\right\|\right)^{p}<\infty .
$$

Now, we have for every non zero $z_{1}, \ldots, z_{n-1} \in X$,

$$
\begin{aligned}
& \left(\left\|\frac{1}{i} \sum_{k=1}^{i} \Delta^{m} x_{k}, z_{1}, \ldots, z_{n-1}\right\|\right) \\
& \quad \leq\left(\left\|\frac{1}{i} \sum_{k=1}^{i} \Delta^{m-1} x_{k}, z_{1}, \ldots, z_{n-1}\right\|\right)+\left(\left\|\frac{1}{i} \sum_{k=1}^{i} \Delta^{m-1} x_{k+1}, z_{1}, \ldots, z_{n-1}\right\|\right) .
\end{aligned}
$$

It is known that for $1 \leq p<\infty$,

$$
|a+b|^{p} \leq 2^{p}\left(|a|^{p}+|b|^{p}\right) .
$$

Hence, for $1 \leq p<\infty$,

$$
\begin{aligned}
& \left(\left\|\frac{1}{i} \sum_{k=1}^{i} \Delta^{m} x_{k}, z_{1}, \ldots, z_{n-1}\right\|\right)^{p} \\
& \quad \leq 2^{p}\left\{\left(\left\|\frac{1}{i} \sum_{k=1}^{i} \Delta^{m-1} x_{k}, z_{1}, \ldots, z_{n-1}\right\|\right)^{p}+\left(\left\|\frac{1}{i} \sum_{k=1}^{i} \Delta^{m-1} x_{k+1}, z_{1}, \ldots, z_{n-1}\right\|\right)^{p}\right\} .
\end{aligned}
$$

Then for each positive integer $r$, we get

$$
\begin{aligned}
& \sum_{i=1}^{r}\left(\left\|\frac{1}{i} \sum_{k=1}^{i} \Delta^{m} x_{k}, z_{1}, \ldots, z_{n-1}\right\|\right)^{p} \\
& \quad \leq 2^{p}\left\{\sum_{i=1}^{r}\left(\left\|\frac{1}{i} \sum_{k=1}^{i} \Delta^{m-1} x_{k}, z_{1}, \ldots, z_{n-1}\right\|\right)^{p}+\sum_{i=1}^{r}\left(\left\|\frac{1}{i} \sum_{k=1}^{i} \Delta^{m-1} x_{k+1}, z_{1}, \ldots, z_{n-1}\right\|\right)^{p}\right\} .
\end{aligned}
$$

Now, as $r \rightarrow \infty$ and using (3.4.1), we have

$$
\sum_{i=1}^{\infty}\left(\left\|\frac{1}{i} \sum_{k=1}^{i} \Delta^{m} x_{k}, z_{1}, \ldots, z_{n-1}\right\|\right)^{p}<\infty .
$$

Thus, $C_{p}\left(\Delta^{m-1},\|\cdot, \ldots, \cdot\|\right) \subset C_{p}\left(\Delta^{m},\|\cdot, \ldots, \cdot\|\right)$ for $1 \leq p<\infty$. The inclusion is strict, and it follows from the following example.

Example 3.1 Let $X=R^{3}$ be a real linear space. Define $\|\cdot, \cdot\|: X \times X \rightarrow R$ by $\|x, y\|=$ $\max \left\{\left|x_{1} y_{2}-x_{2} y_{1}\right|,\left|x_{2} y_{3}-x_{3} y_{2}\right|,\left|x_{3} y_{1}-x_{1} y_{3}\right|\right\}$, where $x=\left(x_{1}, x_{2}, x_{3}\right), y=\left(y_{1}, y_{2}, y_{3}\right)$ are in $R^{3}$. Then $(X,\|\cdot, \cdot\|)$ is a 2-normed linear space. Consider sequence $x=\left\{x_{k}\right\}=\{(k, k, k)\}$ for all $k \in N$. Then $\Delta^{2} x_{k}=(0,0,0)$ for all $k \in N$. Hence, $\left(x_{k}\right) \in C_{p}\left(\Delta^{2},\|\cdot, \cdot\|\right)$. We have $\Delta x_{k}=(-1,-1,-1)$ for all $k \in N$. Hence, $\left(x_{k}\right) \notin C_{p}(\Delta,\|\cdot, \cdot\|)$. Thus, the inclusion is strict. 


\section{Theorem 3.5}

(a) $O_{p}\left(\Delta^{m},\|\cdot, \ldots, \cdot\|\right) \subset C_{p}\left(\Delta^{m},\|\cdot, \ldots, \cdot\|\right) \subset C_{\infty}\left(\Delta^{m},\|\cdot, \ldots, \cdot\|\right)$, and the inclusions are strict.

(b) $O_{p}\left(\Delta^{m},\|\cdot, \ldots, \cdot\|\right) \subset O_{\infty}\left(\Delta^{m},\|\cdot, \ldots, \cdot\|\right) \subset C_{\infty}\left(\Delta^{m},\|\cdot, \ldots, \cdot\|\right)$, and the inclusions are strict.

Proof The proof is easy, so it is omitted.

Remark 3.1 $\ell_{p}\left(\Delta^{m},\|\cdot, \ldots, \cdot\|\right) \not \subset O_{p}\left(\Delta^{m},\|\cdot, \ldots, \cdot\|\right)$. For this, consider the following example.

Example 3.2 Let $p=1$ and a 2-norm $\|\cdot, \cdot\|$ on $X=R^{3}$ as in Example 3.1. Let $m=2$ and consider sequence $\left\{x_{k}\right\}=\{(1,1,1),(0,0,0),(0,0,0),(0,0,0), \ldots)$. Then $\Delta^{2} x_{k}=(1,1,1)$ for $k=$ 1 and $\Delta^{2} x_{k}=(0,0,0)$ for all $k>1$. Then $\left(x_{k}\right) \in \ell\left(\Delta^{2},\|\cdot, \cdot\|\right)$ but $\left(x_{k}\right) \notin O\left(\Delta^{2},\|\cdot, \cdot\|\right)$.

Theorem 3.6 If $1 \leq p<q$, then

(i) $C_{p}\left(\Delta^{m},\|\cdot, \ldots, \cdot\|\right) \subset C_{q}\left(\Delta^{m},\|\cdot, \ldots, \cdot\|\right)$.

(ii) $\ell_{p}\left(\Delta^{m},\|\cdot, \ldots, \cdot\|\right) \subset \ell_{q}\left(\Delta^{m},\|\cdot, \ldots, \cdot\|\right)$.

(iii) $O_{p}\left(\Delta^{m},\|\cdot, \ldots, \cdot\|\right) \subset O_{q}\left(\Delta^{m},\|\cdot, \ldots, \cdot\|\right)$.

Proof The proof is easy, so it is omitted.

\section{Computation of the Köthe-Toeplitz duals}

In order to compute Köthe-Toeplitz dual, we first define the following. An $n$-functional is a real-valued mapping with domain $A_{1} \times \cdots \times A_{n}$, where $A_{1}, \ldots, A_{n}$ are linear manifolds of a linear $n$-normed space.

Let $F$ be an $n$-functional with domain $A_{1} \times \cdots \times A_{n}$. F is called a linear $n$-functional whenever for all ${ }^{1} a_{1},{ }^{1} a_{2}, \ldots,{ }^{1} a_{n} \in A_{1},{ }^{2} a_{1},{ }^{2} a_{2}, \ldots,{ }^{2} a_{n} \in A_{2}, \ldots,{ }^{n} a_{1},{ }^{n} a_{2}, \ldots,{ }^{n} a_{n} \in A_{n}$ and all $\alpha_{1}, \ldots, \alpha_{n} \in R$, we have

(i) $F\left({ }^{1} a_{1}+{ }^{1} a_{2}+\cdots+{ }^{1} a_{n},{ }^{2} a_{1}+{ }^{2} a_{2}+\cdots+{ }^{2} a_{n}, \ldots,{ }^{n} a_{1}+{ }^{n} a_{2}+\cdots+{ }^{n} a_{n}\right)=$ $\sum_{1 \leq i_{1}, i_{2}, \ldots, i_{n} \leq n} F\left({ }^{1} a_{i_{1}},{ }^{2} a_{i_{2}}, \ldots,{ }^{n} a_{i_{n}}\right)$ and

(ii) $F\left(\alpha_{1} a_{1}, \ldots, \alpha_{n} a_{n}\right)=\alpha_{1} \cdots \alpha_{n} F\left(a_{1}, \ldots, a_{n}\right)$.

Let $F$ be an $n$-functional with domain $D(F)$. $F$ is called bounded if there is a real constant $K \geq 0$ such that $\left|F\left(a_{1}, \ldots, a_{n}\right)\right| \leq K\left\|a_{1}, \ldots, a_{n}\right\|$ for all $\left(a_{1}, \ldots, a_{n}\right) \in D(F)$. If $F$ is bounded, we define the norm of $F,\|F\|$ by

$$
\|F\|=\operatorname{glb}\left\{K:\left|F\left(a_{1}, \ldots, a_{n}\right)\right| \leq K\left\|a_{1}, \ldots, a_{n}\right\| \text { for all }\left(a_{1}, \ldots, a_{n}\right) \in D(F)\right\}
$$

If $F$ is not bounded, we define $\|F\|=+\infty$.

It is easy to check the following two results. In this context, one may refer to George [34].

Proposition 4.1 A linear n-functional $F$ is continuous if and only if it is bounded.

Proposition 4.2 Let $B^{*}$ be the set of bounded linear $n$-functionals with domain $B_{1} \times \cdots \times$ $B_{n}$. Then $B^{*}$ is an $n$-Banach space up to linear dependence. 
For any $n(>1)$-normed space $E$, we denote by $E^{*}$ the continuous dual of $E$. There is a need to explore in detail on this notion of continuous duality for $n$-normed spaces.

We have the definition of Köthe-Toeplitz dual of sequence spaces with base space an $n$-normed space as follows.

Let $E$ be an $n$-normed linear space, normed by $\|\cdot, \ldots, \cdot\|_{E}$. Then we define the KötheToeplitz dual of the sequence space $Z(E)$ whose base space is $E$ as

$$
\begin{aligned}
{[Z(E)]^{\alpha}=} & \left\{\left(y_{k}\right): y_{k} \in E^{*}, k \in N \text { and }\left(\left\|x_{k}, u_{2}, \ldots, u_{n}\right\|_{E}\left\|y_{k}, v_{2}, \ldots, v_{n}\right\|_{E^{*}}\right) \in \ell_{1}\right. \\
& \text { for every } \left.v_{2}, \ldots, v_{n} \in E^{*}, u_{2}, \ldots, u_{n} \in E,\left(x_{k}\right) \in Z(E)\right\} .
\end{aligned}
$$

It is easy to check that $\phi \subset X^{\alpha}$. If $X \subset Y$, then $Y^{\alpha} \subset X^{\alpha}$.

Let us consider

$$
S C_{p}\left(\Delta^{m},\|\cdot, \ldots, \cdot\|\right)=\left\{x=\left(x_{k}\right): x \in C_{p}\left(\Delta^{m},\|\cdot, \ldots, \cdot\|\right), x_{1}=\cdots=x_{m}=0\right\} .
$$

Then $S C_{p}\left(\Delta^{m},\|\cdot, \ldots, \cdot\|\right)$ is a subspace of $C_{p}\left(\Delta^{m},\|\cdot, \ldots, \cdot\|\right)$ for $1 \leq p<\infty$. We can have similar subspaces for other spaces as well.

Now, we procure the following results which will be helpful in establishing our result.

Lemma 4.1 (M. Et [35]) $x \in S C_{\infty}\left(\Delta^{m}\right)$ implies that $\sup _{k} k^{-m}\left|x_{k}\right|<\infty$.

Lemma 4.2 $x \in S C_{\infty}\left(\Delta^{m},\|\cdot, \ldots, \cdot\|\right)$ implies that $\sup _{k} k^{-m}\left\|x_{k}, u_{2}, \ldots, u_{n}\right\|<\infty$ for every $u_{2}, \ldots, u_{n} \in X$.

Proof The proof follows using similar techniques as applied in the proof of Lemma 4.1.

Let us set

$$
U=\left\{a=\left(a_{k}\right): \sum_{k=1}^{\infty} k^{m}\left\|a_{k}, z_{2}, \ldots, z_{n}\right\|_{X^{*}}<\infty \text { for every } z_{2}, \ldots, z_{n} \in X^{*}\right\} .
$$

Theorem 4.3 The Köthe-Toeplitz dual of the space $\operatorname{SC}_{p}\left(\Delta^{m},\|\cdot, \ldots, \cdot\|\right)$ is $U$, i.e., $\left[S C_{\infty}\left(\Delta^{m}\right.\right.$, $\|\cdot, \ldots, \cdot\|)]^{\alpha}=U$.

Proof If $a \in U$, then

$$
\begin{aligned}
& \sum_{k=1}^{\infty}\left\|a_{k}, z_{2}, \ldots, z_{n}\right\|_{X^{*}}\left\|x_{k}, u_{2}, \ldots, u_{n}\right\|_{X} \\
& \quad=\sum_{k=1}^{\infty} k^{m}\left\|a_{k}, z_{2}, \ldots, z_{n}\right\|_{X^{*}}\left(k^{-m}\left\|x_{k}, u_{2}, \ldots, u_{n}\right\|_{X}\right) \\
& \quad<\infty,
\end{aligned}
$$

for each $x \in S C_{\infty}\left(\Delta^{m},\|\cdot, \ldots, \cdot\|\right)$ (by Lemma 4.2). Hence, $x \in\left[S C_{\infty}\left(\Delta^{m},\|\cdot, \ldots, \cdot\|\right)\right]^{\alpha}$.

Next, let $a \in\left[S C_{\infty}\left(\Delta^{m},\|\cdot, \ldots, \cdot\|\right)\right]^{\alpha}$. Then $\sum_{k=1}^{\infty}\left\|a_{k}, z_{2}, \ldots, z_{n}\right\|_{X^{*}}\left\|x_{k}, u_{2}, \ldots, u_{n}\right\|_{X}<\infty$ for each $x \in S C_{\infty}\left(\Delta^{m},\|\cdot, \ldots, \cdot\|\right)$. We define sequence $x=\left(x_{k}\right)$ by

$$
x_{k}= \begin{cases}0, & k \leq m, \\ k^{m}, & k>m,\end{cases}
$$


and choose $u_{2}, \ldots, u_{n} \in X$ such that

$$
\left\|k^{m}, u_{2}, \ldots, u_{n}\right\|_{X}=k^{m}\left\|1, u_{2}, \ldots, u_{n}\right\|_{X}= \begin{cases}0, & k \leq m \\ k^{m}, & k>m\end{cases}
$$

We may write for every $z_{2}, \ldots, z_{n} \in X^{*}$,

$$
\begin{aligned}
& \sum_{k=1}^{\infty} k^{m}\left\|a_{k}, z_{2}, \ldots, z_{n}\right\|_{X^{*}} \\
& \quad=\sum_{k=1}^{\infty}\left\|k^{m}, u_{2}, \ldots, u_{n}\right\|_{X}\left\|a_{k}, z_{2}, \ldots, z_{n}\right\|_{X^{*}} \\
& \quad=\sum_{k=1}^{m}\left\|k^{m}, u_{2}, \ldots, u_{n}\right\|_{X}\left\|a_{k}, z_{2}, \ldots, z_{n}\right\|_{X^{*}}+\sum_{k=1}^{\infty}\left\|k^{m}, u_{2}, \ldots, u_{n}\right\|_{X}\left\|a_{k}, z_{2}, \ldots, z_{n}\right\|_{X^{*}} \\
& \quad<\infty .
\end{aligned}
$$

This implies that $a \in U$.

Theorem 4.4 $\left[S C_{\infty}\left(\Delta^{m},\|\cdot, \ldots, \cdot\|\right)\right]^{\alpha}=\left[C_{\infty}\left(\Delta^{m},\|\cdot, \ldots, \cdot\|\right)\right]^{\alpha}$.

Proof Since $S C_{\infty}\left(\Delta^{m},\|\cdot, \ldots, \cdot\|\right) \subset C_{\infty}\left(\Delta^{m},\|\cdot, \ldots, \cdot\|\right)$, we have

$$
\left[C_{\infty}\left(\Delta^{m},\|\cdot, \ldots, \cdot\|\right)\right]^{\alpha} \subset\left[S C_{\infty}\left(\Delta^{m},\|\cdot, \ldots, \cdot\|\right)\right]^{\alpha}
$$

Let $a \in\left[S C_{\infty}\left(\Delta^{m},\|\cdot, \ldots, \cdot\|\right)\right]^{\alpha}$ and $x \in C_{\infty}\left(\Delta^{m},\|\cdot, \ldots, \cdot\|\right)$. If we take sequence $x=\left(x_{k}\right)$ as follows

$$
x_{k}= \begin{cases}x_{k}, & k \leq m, \\ x_{k}^{\prime}, & k>m,\end{cases}
$$

where $x^{\prime}=\left(x_{k}^{\prime}\right) \in S C_{\infty}\left(\Delta^{m},\|\cdot, \ldots, \cdot\|\right)$. Then we may write

$$
\begin{aligned}
& \sum_{k=1}^{\infty}\left\|a_{k}, z_{2}, \ldots, z_{n}\right\|_{X^{*}}\left\|x_{k}, u_{2}, \ldots, u_{n}\right\|_{X} \\
& \quad=\sum_{k=1}^{m}\left\|a_{k}, z_{2}, \ldots, z_{n}\right\|_{X^{*}}\left\|x_{k}, u_{2}, \ldots, u_{n}\right\|_{X}+\sum_{k=1}^{\infty}\left\|a_{k}, z_{2}, \ldots, z_{n}\right\|_{X^{*}}\left\|x_{k}^{\prime}, u_{2}, \ldots, u_{n}\right\|_{X} \\
& \quad<\infty .
\end{aligned}
$$

This implies that $a \in\left[C_{\infty}\left(\Delta^{m},\|\cdot, \ldots, \cdot\|\right)\right]^{\alpha}$.

Theorem $4.5\left[O_{\infty}\left(\Delta^{m},\|\cdot, \ldots, \cdot\|\right)\right]^{\alpha}=\left[C_{\infty}\left(\Delta^{m},\|\cdot, \ldots, \cdot\|\right)\right]^{\alpha}$.

Proof The proof is trivial. 


\section{Competing interests}

The author declares that they have no competing interests.

\section{Acknowledgements}

The research work of this article is supported by the University Grant Commission, New Delhi-110002, India as a minor research project under F. No. 39-935/2010 (SR)

\section{Received: 1 August 2013 Accepted: 12 September 2013 Published: 07 Nov 2013}

\section{References}

1. Zeller, K: Theorie der Limitierungsverfahren. Springer, Berlin (1958)

2. Shive, JS: On the Cesàro sequence spaces. Tamkang J. Math. 1, 19-25 (1970)

3. Siddiqi, AH: 2-normed spaces. Aligarh Bull. Math. 7, 53-70 (1980)

4. Orhan, C: Cesàro difference sequence spaces and related matrix transformations. Commun. Fac. Sci. Univ. Ankara, Sér. A 32, 55-63 (1983)

5. Mursaleen, M, Khatib, MA, Qamaruddin: On difference Cesàro sequence spaces of non-absolute type. Bull. Calcutta Math. Soc. 89, 337-342 (1997)

6. Ng, PN: Matrix transformations on Cesàro sequence spaces of nonabsolute type. Tamkang J. Math. 10, $215-221$ (1979)

7. Ng, PN, Lee, PY: Cesàro sequence spaces of nonabsolute type. Comment. Math. 20, 429-433 (1978)

8. Freedman, AR, Sember, JJ, Raphael, M: Some Cesàro-type summability spaces. Proc. Lond. Math. Soc. 37, 508-520 (1978)

9. Köthe, G, Toeplitz, O: Lineare Räume mit unendlich vielen koordinaten und Ringe unenlicher Matrizen. J. Reine Angew. Math. 171, 193-226 (1934)

10. Lascarides, CG: A study of certain sequence spaces of Maddox and a generalization of a theorem of lyer. Pac. J. Math., 38(2), 487-500 (1971)

11. Maddox, IJ: Continuous and Köthe-Toeplitz duals of certain sequence spaces. Proc. Camb. Philol. Soc. 65, 431-435 (1969)

12. Mursaleen, M, Gaur, AK, Saifi, AH: Some new sequence spaces their duals and matrix transformations. Bull. Calcutta Math. Soc. 88, 207-212 (1996)

13. Gunawan, $\mathrm{H}$ : On $n$-inner product, $n$-norms, and the Cauchy-Schwarz inequality. Sci. Math. Jpn. (Online) 5, 47-54 (2001)

14. Dutta, H: An application of lacunary summability method to $n$-norm. Int. J. Appl. Math. Stat., 15(D09), 89-97 (2009)

15. Gähler, S: Über die Uniformisierbarkeit 2-metrischer Räume. Math. Nachr. 28, 235-244 (1964)

16. Gähler, S: Über 2-Banach Räume. Math. Nachr. 42, 335-347 (1969)

17. Gähler, S: Untersuchungen über verallgemeinerte m-metrische Räume, II. Math. Nachr. 40, 229-264 (1969)

18. Gähler, S, Siddiqi, AH, Gupta, SC: Contributions to non-Archimedean functional analysis. Math. Nachr. 69, 162-171 (1975)

19. Gunawan, H: The space of p-summable sequences and its natural n-norm. Bull. Aust. Math. Soc., 64(1), 137-147 (2001)

20. Gunawan, H, Mashadi, M: On finite dimensional 2-normed spaces. Soochow J. Math., 27(3), 631-639 (2001)

21. Gunawan, H, Mashadi, M: On n-normed spaces. Int. J. Math. Math. Sci 27(10), 631-639 (2001)

22. Dutta, H: On n-normed linear space-valued null, convergent and bounded sequences. Bull. Pure Appl. Math. 4(1) 103-109 (2010)

23. Kim, SS, Cho, YJ: Strict convexity in linear n-normed spaces. Demonstratio Math 29, 739-744 (1996)

24. Misiak, A: n-inner product spaces. Math. Nachr. 140, 299-319 (1989)

25. Misiak, A: Orthogonality and orthonormality in $n$-inner product spaces. Math. Nachr. 143, $249-261$ (1989)

26. Malèeski, A: Strong n-convex n-normed spaces. Mat. Bilt. 21, 81-102 (1997)

27. Diminnie, C, Gähler, S, White, A: 2-inner product spaces. Demonstrario Math. 6, 525-536 (1973)

28. Gähler, S: 2-metrische Räume und ihre topologische Struktur. Math. Nachr. 26, 115-148 (1963)

29. Gähler, S: 2-normed spaces. Math. Nachr. 28, 1-43 (1964)

30. Gähler, S: Lineare 2-normierte Räume. Math. Nachr. 28, 1-43 (1964)

31. Kizmaz, H: On certain sequence spaces. Can. Math. Bull. 24(2), 169-176 (1981)

32. Et, M, Colak, R: On generalized difference sequence spaces. Soochow J. Math., 21(4), 377-386 (1995)

33. Tripathy, BC, Esi, A, Tripathy, BK: On a new type of generalized difference Cesàro Sequence spaces. Soochow J. Math., 31(3), 333-340 (2005)

34. George, WA Jr.: 2-Banach spaces. Math. Nachr. 42, 43-60 (1969)

35. Et, M: On some generalized Cesàro difference sequence spaces. Istanbul Univ. fen fak. Mat. Dergisi, 55-56, 221-229 (1996-1997)

10.1186/1687-1847-2013-286

Cite this article as: Dutta: Some classes of Cesàro-type difference sequences over $n$-normed spaces. Advances in Difference Equations 2013, 2013:286 